\title{
Design, analysis, and feedback control of a nonlinear micro piezoelectric-electrostatic energy harvester
}

\author{
S. Amir Mousavi Lajimi* • Michael I. Friswell
}

Received: date / Accepted: date

\begin{abstract}
A nonlinear micro piezoelectric-electrostatic energy harvester is designed and studied using mathematical and computational methods. The system consists of a cantilever beam substrate, a bimorph piezoelectric transducer, a pair of tuning parallel-plate capacitors, and a tip-mass. The governing nonlinear mathematical model of the electro-mechanical system including nonlinear material and quadratic air-damping is derived for the series connection of the piezoelectric layers. The static and modal frequency curves are computed to optimize the operating point, and a parametric study is performed using numerical methods. A bias DC voltage is used to adapt the system to resonate with respect to the frequency of external vibration. Furthermore, to improve the bandwidth and performance of the harvester (and achieve a high level of harvested power without sacrificing the bandwidth) a nonlinear feedback loop is integrated into the design.
\end{abstract}

Keywords Energy Generation · Nonlinearity · Piezoelectric · Electrostatic · Feedback control . Performance

\section{Introduction}

Sensors and actuators have been developed using microelectromechanical systems (MEMS) and powered by external (on board) power sources. As a result, the weight of these systems increases as the power source needs to

S. Amir Mousavi Lajimi (Corresponding author)

University of Waterloo, Waterloo N2L 3G1, Canada

E-mail: amir.lajimi@uwaterloo.ca, samousav@uwaterloo.ca

Michael I. Friswell

Swansea University, Swansea SA1 8EN, UK

E-mail:m.i.friswell@swansea.ac.uk maintain a certain capacity. Therefore inventing selfpowered MEMS devices is strongly desirable especially for medical applications. Vibration energy harvesting systems may be miniaturized sufficiently to be integrated into micro-electromechanical systems (MEMS) to produce self-powered wireless sensors [10,42]. Hence, many researchers are working on energy harvesting systems at the micro-scale.

Various structures and transduction methods are used to convert the vibratory energy into electric energy [4,6]. Arnold [3] presented a comprehensive review of magnetic power generation at the micro-scale, Mitcheson et al. [29] described an electrostatic microcapacitor for power generation, and Liu et al. [24] proposed a piezoelectric energy harvester. The reviews by Beeby et al. [4] and Cook et al. [6] revealed that piezoelectric energy harvesting is employed more than any other transduction mechanism. There is also an ongoing investigation into various structural configurations and excitation mechanisms to realize an optimum (nonlinear) harvester. Leadenham and Erturk [21] presented an M-shaped oscillator for broadband energy harvesting and Lee et al. [23] introduced a zigzag shaped torsional energy harvester. Benefiting from the asymmetric nonlinear behaviour of the harvester, a broadband behaviour was demonstrated under various excitation levels. Cantilever structures are most commonly used, and the early works by Jeon et al. [13] and Beeby et al. [4] presented different cantilever structures for energy harvesting. More recently, Jia and Seshia [15] studied different cantilever structures, some as long as $2000 \mu \mathrm{m}$, and showed that the length of the micro-structure plays a significant role in the power capacity of the structure.

A major challenge in designing energy harvesting systems at both the macro- and micro-scale is to optimize the performance of the system. Although the fre- 
quency content of environmental vibration is often scattered over a wide range, the harvesting devices do not generate their peak power for a such large range of input excitation. One approach to overcome this challenge is to design an adaptive system. To this end, Madinei et al. $[25,26]$ performed a preliminary study of a micro energy harvester with parallel piezo layers benefiting from tuning via an electrostatic actuator. A solution to improve energy harvesting under wide-spectrum vibration is to create a bistable system oscillating between the two stable states. Stanton et al. [39] and Ferrari et al. [9] designed cantilever beams carrying end magnets operating in the nonlinear regime and demonstrated bistable responses. Stanton et al. [38] examined a different configuration using a pair of external magnets to increase the bandwidth of the harvester. Kumar et al. [17] proposed to use an asymmetric monostable to overcome the high activation threshold of bistable harvesters. Alevras et al. discussed the details of the nonlinear dynamics of a magnetic levitation harvester and showed that the additional resonant zones enhances the energy harvesting capabilities of the harvester [2].

The excitation mechanism plays a significant role in the system response and consequently in the efficiency of power generation. Direct and parametric resonances demonstrate different characteristics and therefore are desired for different reasons. Using a perturbation approach Daqaq et al. [7] analyzed a cantilever beam under base excitation and presented parametric resonance as a viable option for energy harvesting. Abdelkafi et al. [1] presented a theoretical analysis of a cantilever beam experiencing parametric resonance and briefly discussed the effect of a quadratic damping term on the behaviour of the reduced-model of the harvester. Auto-parametric resonance as well as parametric resonance demonstrates a promising potential for energy harvesting for real applications [14]. Although parametric resonance, in contrast to direct resonance, is not limited by linear damping, its onset of activation is negatively affected by increasing linear damping [16]. Therefore, ongoing research is investigating methods to reduce the activation threshold. More recently, to simultaneously attack the shortcomings of conventional energy harvesters, Hwang and Arrieta [12] proposed to use 1D lattices of bistable elements. The proposed complex design demonstrated promise.

To explain the bandwidth and amplitude requirements, we provide an insight into the physics of the harvester. The underlying oscillator is initiated under external excitation and the oscillation amplitude increases once the energy accumulates above a certain threshold. Various mechanisms provide damping and extract the energy from the system, which negatively affects the amplitude and bandwidth of the harvester. Developing self-sustaining oscillators is currently investigated by different researchers, e.g. see [5] and the references there. However, the presence of a feedback loop drawing power from the harvester (or an external source) is not desired. Different methods have been proposed and are under consideration to improve the bandwidth and signal-to-noise ratio of nonlinear resonators by various research groups $[18,20,33,34,35,41]$.

In this paper, we present the analysis and design of a micro piezoelectric-electrostatic energy harvester. The piezoelectric transduction mechanism is complemented with an electrostatic tuning mechanism. Using a symmetric set of electrostatic electrodes provides a means to adjust the effective natural frequency of the system with minimum interference of the initial undeformed configuration of the structure. The model of the system is developed using an energy approach for a series connection of piezoelectric layers; the extension to a parallel connection is straight forward. Considering the effects of varying base-frequency, base-acceleration, nonlinear damping, and electrical load resistance, a comprehensive parametric study of the system is performed. The optimum electrical load resistance for maximum power output is computed and shown to depend on the base-acceleration level. We design a nonlinear feedback loop to further sustain the oscillations, increase the bandwidth, improve the performance of the harvester, and increase the average harvested power.

\section{Model of the energy harvester}

The cantilever beam (substrate) carries a tip-mass enabling an initial tuning of the mechanical resonance. An energy-based approach is employed to develop the (mathematical) model of the system. The potential energy of the system includes the elastic energy of the substrate and the piezoelectric layer(s), the electrostatic potential energy due to the varying gap parallel-plate capacitors, and the electric enthalpy of piezoelectric material. The kinetic energy of the system is developed for the moving substrate, the end-mass, and the piezoelectric layer, although the latter is negligible due to the relatively small thickness of the layer. The virtual nonconservative work represents the linear and nonlinear mechanical damping and the energy dissipation through the electrical load resistance (the harvester).

The structural, material, and electrical properties of the micro-harvester are given in Tables 1 and 2. The effective Young's modulus is $E_{s}=\hat{E}_{s} /\left(1-\nu_{s}^{2}\right)\left(\nu_{s}=\right.$ 0.06 represents Poisson's ratio) given that the thickness of the substrate (beam) is significantly smaller than its width [32]. 
Table 1: Structural Properties $(\mu \mathrm{m})$

\begin{tabular}{|llllll|}
\hline \hline$L_{s}$ & 2500 & $L_{p}$ & 2500 & $L_{m}$ & 750 \\
$h_{s}$ & 5 (along z-axis) & $h_{p}$ & 2 & $h_{m}$ & 250 \\
$b_{s}$ & 500 (along y-axis) & $b_{p}$ & 500 & $b_{m}$ & 500 \\
\hline
\end{tabular}

Table 2: Material and Electrical Properties

\begin{tabular}{|ll|}
\hline \hline$\rho_{s}$ & $2330 \mathrm{~kg} / \mathrm{m}^{3}$ \\
$\rho_{p}$ & $7800 \mathrm{~kg} / \mathrm{m}^{3}$ \\
$\rho_{m}$ & $2330 \mathrm{~kg} / \mathrm{m}^{3}$ \\
$\hat{E}_{s}$ & $169 \mathrm{GPa}$ \\
$\nu_{s}$ & 0.06 \\
$c_{p}^{11}$ & $72 \mathrm{GPa}$ \\
$c_{p}^{1111}$ & $-72 \times 10^{7} \mathrm{GPa}$ \\
$\epsilon_{r}$ & $1 \mathrm{~F} / \mathrm{m}$ \\
$\epsilon_{0}$ & $8.854 \times 10^{-12} \mathrm{~F} / \mathrm{m}$ \\
$\epsilon_{33}$ & $8.854 \times 10^{-9} \mathrm{~F} / \mathrm{m}$ \\
$e_{31}$ & $-11.6 \mathrm{C} / \mathrm{m}^{2}$ \\
$e_{3111}$ & $-11.6 \times 10^{7} \mathrm{C} / \mathrm{m}^{2}$ \\
\hline
\end{tabular}

The axial deformation (extension) of the cantilever is characterized using the inextensibility principle. While the stress-strain relationship is linear, the axial strain caused by the transverse deformation, $w(r, t)$ where $r$ is the position on the beam, is modeled using a cubic function of the displacement. Therefore the axial stress and strain are [30],

$$
\begin{aligned}
T_{r}(r, z, t) & =E_{s} S_{r}(r, z, t) \\
S_{r}(r, z, t) & =-z \kappa(r, t) \\
\kappa(r, t) & \approx w(r, t)_{, r, r}\left(1+\frac{1}{2} w(r, t)_{, r}^{2}\right) \\
\phi(r, t) & =\arcsin \left(w(r, t)_{, r}\right) \\
& \approx w(r, t)_{, r}+\frac{1}{6} w(r, t)_{, r}^{3}
\end{aligned}
$$

where $\kappa(r, t)$ and $\phi(r, t)$ indicate the curvature and slope of the beam at $r$ and $(\cdot)_{, r}$ and $(\cdot)_{, r, r}$ the first and second partial differentiation with respect to the $r$. A schematic of the micro-system for energy harvesting is shown in Figure 1. For the series connection, the piezoelectric layers are polarized in opposite directions [40]; the parallel connection, where the polarization is in the same direction, is easily modeled if required. Thus, the transverse electric fields in the piezoelectric layers connected in series are modeled as

$E_{3}^{u}(r, t)=\frac{1}{2 h_{p}} \lambda(r, t)_{, t} \quad E_{3}^{l}(r, t)=-\frac{1}{2 h_{p}} \lambda(r, t)_{, t}$

where $\lambda$ is the electric flux linkage coordinate $[39,22]$ and $(\cdot)_{, t}$ the first partial differentiation with respect to time $t$. Superscripts $u$ and $l$ indicate the two piezoelectric layers. Given that strains above and below the neutral axis have opposite signs, poling the two layers oppositely results in generating electric fields in the

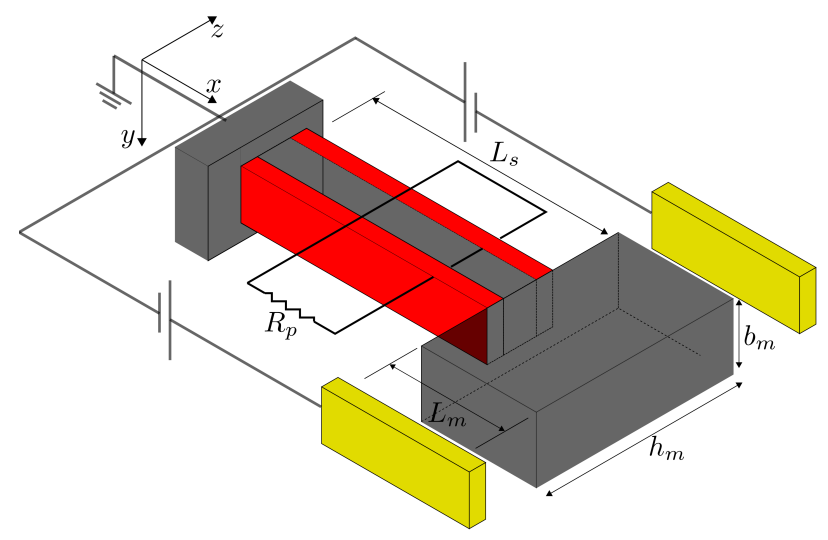

Fig. 1: The schematic of the harvester under direct base-excitation. The figure is not drawn to scale.

same direction [8]. The potential energy of the beam is given by

$\mathcal{P}_{s}=\frac{1}{2} E_{s} I_{s} \int_{0}^{L_{s}} \kappa(r, t)^{2} \mathrm{~d} r$

and the electric enthalpy by $[22,37,39]$

$$
\begin{aligned}
\mathcal{P}_{p}=\int_{0}^{b} & \int_{\frac{h_{s}}{2}}^{h_{p}+\frac{h_{s}}{2}} \\
& \int_{0}^{L_{p}}\left(\frac{1}{2} c_{p}^{11} S_{r}(r, z, t)^{2}+\frac{1}{3} c_{p}^{111} S_{r}(r, z, t)^{3}\right. \\
& +\frac{1}{4} c_{p}^{1111} S_{r}(r, z, t)^{4}-e_{31} E_{3}^{l}(r, t) S_{r}(r, z, t) \\
& +\frac{1}{2} e_{311} E_{3}^{l}(r, t) S_{r}(r, z, t)^{2} \\
& -\frac{1}{3} e_{3111} E_{3}^{l}(r, t) S_{r}(r, z, t)^{3} \\
& \left.-\frac{1}{2} \epsilon_{33} E_{3}^{l}(r, t) E_{3}^{l}(r, t)\right) \mathrm{d} r \mathrm{~d} z \mathrm{~d} y \\
+ & \int_{0}^{b}+\frac{h_{s}}{2} \\
& \int_{0}^{L_{p}}\left(\frac{1}{2} c_{p}^{11} S_{r}(r, z, t)^{2}+\frac{1}{3} c_{p}^{111} S_{r}(r, z, t)^{3}\right. \\
& +\frac{1}{4} c_{p}^{1111} S_{r}(r, z, t)^{4}-e_{31} E_{3}^{l}(r, t) S_{r}(r, z, t) \\
& +\frac{1}{2} e_{311} E_{3}^{u}(r, t) S_{r}(r, z, t)^{2} \\
& -\frac{1}{3} e_{3111} E_{3}^{u}(r, t) S_{r}(r, z, t)^{3} \\
& \left.-\frac{1}{2} \epsilon_{33} E_{3}^{u}(r, t) E_{3}^{u}(r, t)\right) \mathrm{d} r \mathrm{~d} z \mathrm{~d} y
\end{aligned}
$$

Meijs and Fokkema [27] discussed the importance of fringing fields in parallel-pate capacitors and presented 
an accurate model of the varying parallel-plate capacitor including the fringing capacitance. Using their capacitance model, the electrical potential energy is [11]

$$
\begin{aligned}
\mathcal{P}_{e} & =-\frac{1}{2} \epsilon_{o} \epsilon_{r} \int_{r_{1}}^{r_{2}} V_{\mathrm{es}}(t)^{2}\left(1.54+\frac{b_{s}}{g_{o}^{2}-w(r, t)^{2}}\right. \\
& +\left(\frac{1.2625 b_{s}}{g_{o}-w(r, t)}\right)^{0.25}+\left(\frac{1.2625 b_{s}}{g_{o}+w(r, t)}\right)^{0.25} \\
& \left.+\left(\frac{1.1236 h_{s}}{g_{o}-w(r, t)}\right)^{0.5}+\left(\frac{1.1236 h_{s}}{g_{o}+w(r, t)}\right)^{0.5}\right) \mathrm{d} r
\end{aligned}
$$

Expanding the capacitance terms about $w_{s}(r, t)=0$, where $w(r, t)=w_{s}(r, t)+w_{d}(r, t)$, using a Taylor's series and truncating the fifth and higher order terms we obtain an approximate electrostatic potential energy expression. To this end, the dynamic transverse displacement along the length of the end rigid-body is described in the form of $w_{d}(r, t)=w_{d}\left(L_{s}, t\right)+r w_{d}^{\prime}\left(L_{s}, t\right)$, where $w_{d}\left(L_{s}, t\right)$ and $w_{d}^{\prime}\left(L_{s}, t\right)$ represent the transverse displacement and slope at the beam's tip. The distance variable $r$ in this relationship varies between zero and the length of the end rigid-body. Note that the symmetric configuration of electrodes eliminates the effect of odd terms in the electrostatic energy expression.

The energy of the system is mainly dissipated through the linear and nonlinear structural damping, the nonlinear air damping, and the electrical load. The variation of nonconservative work is described by

$$
\begin{aligned}
\delta W_{n c}= & -\int_{0}^{L_{s}}\left[c_{l} w(r, t)_{, t}-c_{n l} w(r, t)_{, t} w(r, t)^{2}\right. \\
& \left.-c_{a} w(r, t)_{, t}\left|w(r, t)_{, t}\right|\right] \delta w(r, t) \mathrm{d} r \\
& -\frac{1}{R_{p}} \lambda(r, t)_{, t} \delta \lambda(r, t)
\end{aligned}
$$

\subsection{Kinetic energy}

The kinetic energy is due to the distributed mass of the beam (substrate), the distributed mass of the piezoelectric layers, and the tip-mass. Assuming a uniform mass per unit length along the substrate and the piezoelectric layers $\left(m_{s}\right.$ and $\left.m_{p}\right)$ and including the mass $\left(m_{t}\right)$ and rotary inertia $\left(J_{t}\right)$ of the tip-mass, the kinetic energy expression is, for base excitation $z(t)$,

$$
\begin{aligned}
\mathcal{K}_{s}= & \frac{1}{2} \int_{0}^{L_{s}} \rho_{s} A_{s}(w(r, t)+z(t))_{, t}^{2}+u(r, t)_{, t}^{2} \mathrm{~d} r \\
& +\frac{1}{2} \int_{0}^{L_{p}} \rho_{p} A_{p}\left((w(r, t)+z(t))_{, t}^{2}+u(r, t)_{, t}^{2}\right) \mathrm{d} r \\
& +\frac{1}{2} m_{t}\left(\left(w\left(L_{s}, t\right)+z(t)\right)_{, t}^{2}+u\left(L_{s}, t\right)_{, t}^{2}\right)
\end{aligned}
$$

$$
+\frac{1}{2} J_{t} \phi\left(L_{s}, t\right)_{, t}^{2}
$$

\section{Reduced-order model of energy harvester}

As mentioned earlier, the neutral axis of the beam is inextensional. That is $[36,31]$

$$
\begin{aligned}
\left(1+u(r, t)_{, r}\right)^{2}+w(r, t)_{, r}^{2}=1 \\
\Rightarrow u(r, t)=\int_{0}^{r}\left(\sqrt{1-w(r, t)_{, r}^{2}}-1\right) \mathrm{d} r \\
u(r, t) \approx-\frac{1}{2} \int_{0}^{r} w(r, t)_{, r}^{2} \mathrm{~d} r
\end{aligned}
$$

and

$u(r, t)_{, t} \approx-\frac{1}{2} \frac{\mathrm{d}}{\mathrm{d} t} \int_{0}^{r} w(r, t)_{, r}^{2} \mathrm{~d} r$

Substituting Equations (2) - (6) and (13) into (8)-(11), the mathematical model (i.e. the differential equations) describing the response of the system is obtained using the Rayleigh-Ritz method (or the method of assumed modes) [28]. A modal expansion of the structure's response (the deflection of the structure) is described by

$w(r, t)=\sum_{n=1}^{N} \psi_{n}(r) q_{n}(t)$

where $N$ is the number of linear modes, $\psi_{n}(r)$. The linear modes are obtained by solving the linear eigenvalue problem for a clamped-free beam carrying the tip-mass and can be found in various references, e.g. [19]. Substituting Equation (14) into energy and non-conservative work expressions, and applying Lagrange's equations [28] results in the governing differential equations. Here, a single mode approximation is used.

The Lagrangian of the system, expressed in the form $\mathcal{L}=\mathcal{K}_{s}-\mathcal{P}_{s}-\mathcal{P}_{p}-\mathcal{P}_{e}$, is substituted into the EulerLagrange equations,

$\frac{\mathrm{d}}{\mathrm{d} t} \frac{\partial \mathcal{L}}{\partial \dot{q}_{i}}-\frac{\partial \mathcal{L}}{\partial q_{i}}=Q_{i}$

where $i=1,2$ and $q_{1}=q(t), q_{2}=\lambda(t)$. The generalized forces are

$Q_{q}=-c_{l} \dot{q}(t)-c_{n l} q(t)^{2} \dot{q}(t)-c_{a} \dot{q}(t)|\dot{q}(t)|$

$Q_{\lambda}=\frac{1}{R_{p}} \lambda(r, t)_{, t}$

where $\dot{q}$ represents the time-derivative of the modalcoordinate, $q$. The time-derivatives of flux linkage, $\dot{\lambda}$ and $\ddot{\lambda}$, are replaced with voltage $V_{p}(t)$ and its derivative $\dot{V}_{p}(t)$, respectively, to obtain, by discarding higher than third-order terms, 


$$
\begin{aligned}
\left(m_{l}+m_{n} q(t)^{2}\right) \ddot{q}(t)+m_{n} q(t) \dot{q}(t)^{2} & \\
& +\left(k_{l}-\gamma_{l} V_{\mathrm{es}}(t)^{2}+2 h_{l}^{q}\right) q(t) \\
& +\left(c_{l}+c_{n} q(t)^{2}+c_{a}|\dot{q}(t)|\right) \dot{q}(t) \\
& +\left(2 k_{n}+4 h_{n}^{q}-2 \gamma_{n} V_{\mathrm{es}}(t)^{2}\right) q(t)^{3} \\
& +\left(h_{l}^{\lambda}+3 h_{n}^{\lambda} q(t)^{2}\right) V_{p}(t)=-f_{l} \ddot{F}(t) \\
2 C_{p} \dot{V}_{p}(t) & +\frac{V_{p}(t)}{R_{p}}=\left(h_{l}^{\lambda}+3 h_{n}^{\lambda} q(t)^{2}\right) \dot{q}(t)
\end{aligned}
$$

The preceding equation describes the coupled electromechanical model of a nonlinear piezoelectric-electrostatic energy harvester including nonlinear damping, inertial, stiffness, forcing, and energy harvesting terms. The coefficient parameters are introduced in the Appendix.

\section{Static and modal analysis}

To compute the static fixed (equilibrium) points of (18)(19), the velocity and acceleration terms are set to zero, therefore at the equilibrium point

$\ddot{q}(t) \equiv 0, \dot{q}(t) \equiv 0, \dot{V}_{p}(t) \equiv 0$

resulting in $V_{p}(t) \equiv 0$. The nontrivial (static) equilibrium points are found by solving

$$
\begin{aligned}
& \left(2 k_{n}+4 h_{n}^{q}-2 \gamma_{n} V_{\mathrm{es}}^{2}\right) q_{s}^{2} \\
& +\left(k_{l}-\gamma_{l} V_{\mathrm{es}}^{2}+2 h_{l}^{q}\right)=0
\end{aligned}
$$

Therefore there are two equilibrium points (other than $\left.q_{s}=0\right)$ for

$2 k_{n}+4 h_{n}^{q}-2 \gamma_{n} V_{\mathrm{es}}^{2} \neq 0$

and

$-\left(k_{l}-\gamma_{l} V_{\mathrm{es}}^{2}+2 h_{l}^{q}\right) /\left(2 k_{n}+4 h_{n}^{q}-2 \gamma_{n} V_{\mathrm{es}}^{2}\right)>0$.

For the proposed design in Table 1, the static equilibrium points are computed and plotted in Figure 2 and the bifurction point where the nontrivial equilibrium points disappear are computed to be $23.986,36.619$, and 59.430 Volts for $h_{p}=0.5 \mu \mathrm{m}, h_{p}=2 \mu \mathrm{m}$, and $h_{p}=4 \mu \mathrm{m}$, respectively. Representing the system in (18)-(19) in state-space form and expanding and linearizing the solution around the equilibrium, the Jacobian matrix is found and used to identify the stability of each branch. For small vibration around any static equilibrium, the equation of motion is linearized by substituting $q(t)=q_{s}+q_{d}(t)$ and Equation (20) into the

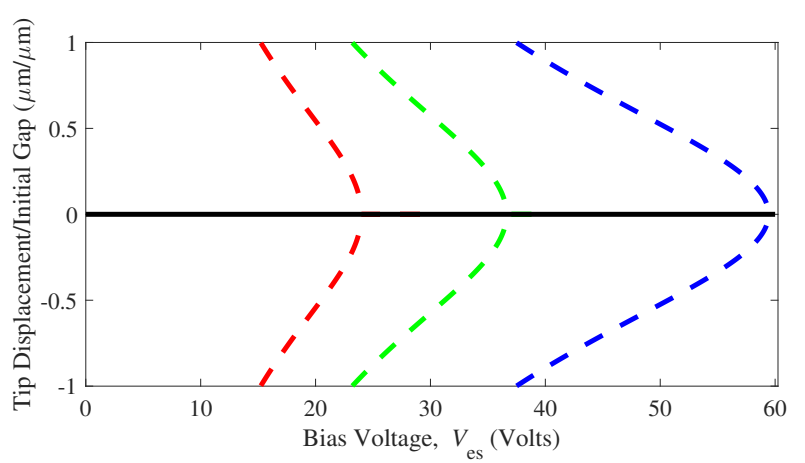

Fig. 2: Static equilibrium points. The horizontal black line indicates the trivial stable equilibrium. (Red: $h_{p}=$ $0.5 \mu \mathrm{m}$, Green: $h_{p}=2 \mu \mathrm{m}$, Blue: $\left.h_{p}=4 \mu \mathrm{m}\right)$

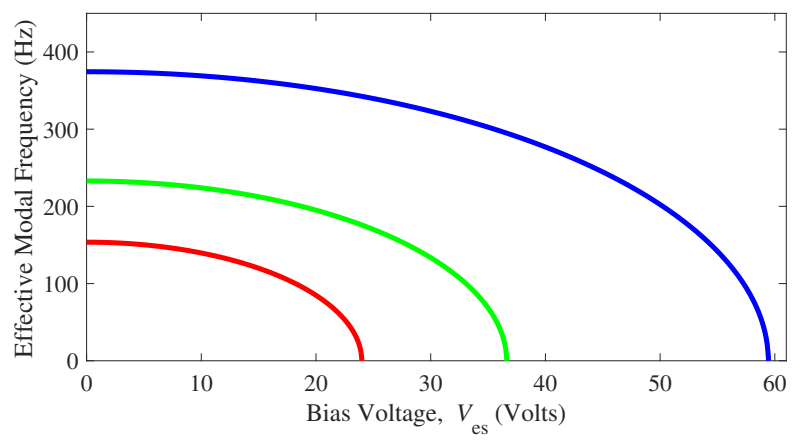

Fig. 3: Effective modal frequency around the undeformed configuration for $h_{s}=4 \mu \mathrm{m}, g_{o}=30 \mu \mathrm{m}$. (Red: $h_{p}=0.5 \mu \mathrm{m}$, Green: $h_{p}=2 \mu \mathrm{m}$, Blue: $\left.h_{p}=4 \mu \mathrm{m}\right)$.

equation of motion. Thus

$$
\begin{aligned}
& \left(m_{l}+m_{n} q_{s}^{2}\right) \ddot{q}_{d}(t) \\
& +\left(2 h_{l}^{q}+q_{s}^{2}\left(12 h_{n}^{q}+6 k_{n}-6 \gamma_{n} V_{\mathrm{es}}^{2}\right)+k_{l}-\gamma_{l} V_{\mathrm{es}}^{2}\right) \\
& \times q_{d}(t)=0
\end{aligned}
$$

Therefore, the linearized, undamped, unforced equation of motion around the trivial static equilibrium $\left(q_{s}=0\right)$ is given by

$m_{l} \ddot{q}_{d}(t)+\left(2 h_{l}^{q}+k_{l}-\gamma_{l} V_{\mathrm{es}}^{2}\right) q_{d}(t)=0$

and the small vibration frequency is

$\omega_{e}^{2}=\frac{\left(k_{l}-\gamma_{l} V_{\mathrm{es}}^{2}+2 h_{l}^{q}\right)}{m_{l}}$

The thickness of piezoelectric harvester affects both the stationary points and the effective modal frequency. Figure 3 shows the variation in effective small vibration (natural) frequency as the DC voltage is increased. 


\section{Dynamic and nonlinear analysis}

To compute the response characteristic, the steady-state response is sought and computed by integrating equations of motion for a sufficiently large time-span. For this particular design and integration method, the governing equations, equations (18)-(19), the equation of motion is initially integrated for 400 cycles, and the response is sampled for the last period. To further follow the steady state solution the variable of interest (base frequency/base amplitude/circuit resistance) is varied (increased/decreased) and the next solution is computed. The amplitude is estimated by taking the absolute average of the difference between the maximum and minimum of the response.

The quality factor is set to 100 (equivalent to $c_{l}=$ $5.839 \times 10^{-3} \mathrm{Ns} / \mathrm{m}$ ) and the electrical load resistance in the harvesting circuit is initially set to $R_{p}=1 \mathrm{M} \Omega$. The base acceleration is described in the form $\ddot{F}(t)=$ $a_{b} \cos \left(2 \pi \omega_{b} t\right)$ where $a_{b}$ and $\omega_{b}$ indicate the base- acceleration and the base-frequency, respectively. In all figures, the forward-sweep curve is indicated with a thicker line than the backward-sweep curve to further clarify the jump phenomena in the response.

Figures $4 \mathrm{a}$ to $4 \mathrm{~d}$ show the amplitude of tip displacement (the frequency-response curve), the phaseportrait at $\omega_{b}=205.7 \mathrm{~Hz}$, the normalized RMS of voltage drop across the resistor, and the normalized average harvested power, respectively, for a varying baseexcitation frequency. Nevertheless, the system is able to safely harvest up to $18 \mathrm{nW} / \mathrm{g}$ along the stable dynamic equilibrium. Note that the resistance of the harvesting circuit is set to $1 \mathrm{M} \Omega$, the $\mathrm{DC}$ voltage to $10 \mathrm{~V}$, the quality factor to 100 , and the material-damping equal to zero. The coefficient of modal air damping $c_{a}$ is increased from 0 (red curve) to 15 (blue curve), 30 (green curve), and to 45 (black curve) $\mathrm{Ns}^{2} / \mathrm{m}^{2}$ and all four sets of curves are plotted in Figures $4 \mathrm{a}$ to $4 \mathrm{~d}$. By increasing the air damping the jump frequencies decrease and move to the right. Once the air damping is large enough the response becomes linear and single-valued (the forward- and backward-sweep curves completely overlap).

Figures $5 \mathrm{a}$ to $5 \mathrm{~d}$ show the normalized amplitude of tip displacement (or the frequency-response curve), the normalized RMS of displacement, the normalized RMS of voltage drop across the resistor, and the normalized average harvested power, respectively, for a varying base-excitation frequency as the nonlinear damping coefficient is increased. Note that the resistance of the harvesting circuit is set to $1 \mathrm{M} \Omega$, the $\mathrm{DC}$ voltage to $10 \mathrm{~V}$, the quality factor to 100 , and the air-damping equal to zero. While the cubic nonlinearity affects the unstable equilibrium points, it does not affect the stable equilibrium points (the thick line). Therefore, practically speaking it would be beneficial to increase the cubic nonlinear damping while keeping the air damping to a minimum.

Figures $6 \mathrm{a}$ to $6 \mathrm{~d}$ show the amplitude of the tipdisplacement, the RMS of the tip-displacement, the average harvested power, and the amplitude of voltage drop across the resistor, for a varying base-excitation amplitude. Figures 6e and $6 \mathrm{f}$ show the phase-portrait (both stable and unstable orbits) for two sets of airdamping and base-excitation parameters. The modal air damping $c_{a}$ is increased from 0 to 15,30 , and to $45 \mathrm{Ns}^{2} / \mathrm{m}^{2}$ and all four sets of curves are plotted in red, blue, green, and black colors, respectively. Similar to frequency-response curves in Figures 4a to 4d, increasing the quadratic (air) damping suppresses the multi-valued response and once the air damping is large enough the response becomes linear and single-valued.

The harvesting circuit plays a crucial role in optimizing the output of the device. We have employed a simple circuit to harvest the vibration energy, however other components, such as capacitors and inductances, may be introduced into the circuit to further meet the requirements of the application. Nevertheless, in this simple circuit optimizing the resistance is important. To demonstrate the significance of a varying resistance, the load resistance $\left(R_{p}\right)$ is varied between $10 \mathrm{k} \Omega$ and $1000 \mathrm{k} \Omega$ and the response is computed for three cases where the DC voltage is increased from 5, to 10, and $15 \mathrm{~V}$, and the base excitation frequency and acceleration are set to $\omega_{b}=225 \mathrm{~Hz}$ and $a_{b}=0.12 \mathrm{~g}$, respectively. The quadratic and cubic damping coefficients are set to zero.

In Figures 7a-7d, we plot the normalized response of the harvester. As the DC (tuning) voltage is increased the optimal resistance for maximum power increases from $35.9 \mathrm{k} \Omega$, to $129 \mathrm{k} \Omega$, and finally to $572 \mathrm{k} \Omega$. The larger the tuning DC voltage is, the larger the resistance needs to be for the peak voltage to approach a relatively constant value. For $V_{\mathrm{es}}=5 \mathrm{~V}$, the response is nonlinear and multivalued between $21.7 \mathrm{k} \Omega$ and $35.9 \mathrm{k} \Omega$. The maximum scavenged power occurs at $R_{p}=35.9 \mathrm{k} \Omega$ and is equal to $20.88 \mathrm{nW} / \mathrm{g}$. To further reveal the details of the transition of the response, in Figure $7 \mathrm{c}$ we plot the variation of RMS Voltage for a smaller resistance range. The resolution of each curve is $100 \Omega$.

\section{Nonlinear feedback mechanism}

To improve the performance of the energy harvester, a feedback is designed and a nonlinear signal proportional to $q(t) \dot{q}(t)^{2}$ is fed back into the energy harvester, see 


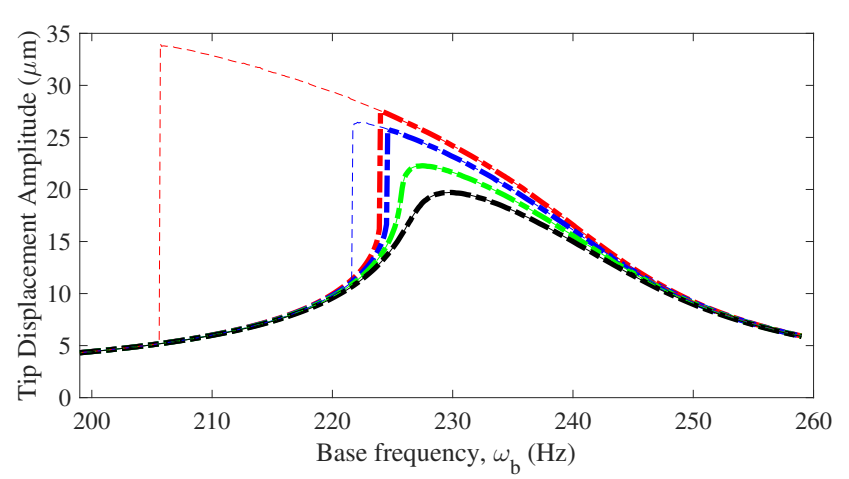

(a) Tip Displacement Amplitude

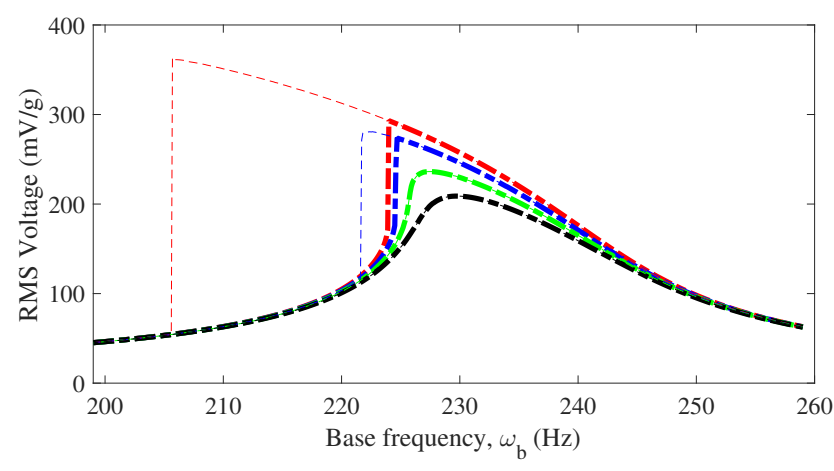

(c) Normalized Voltage

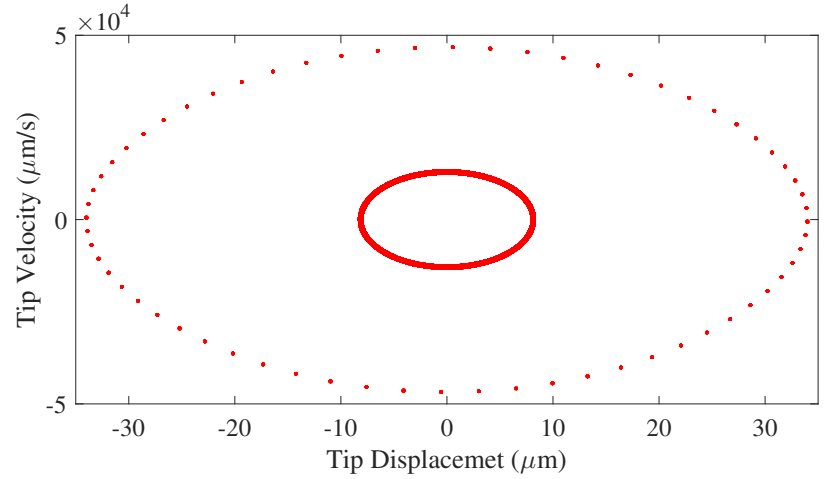

(b) Phase Portrait at $\omega_{b}=205.7 \mathrm{~Hz}$

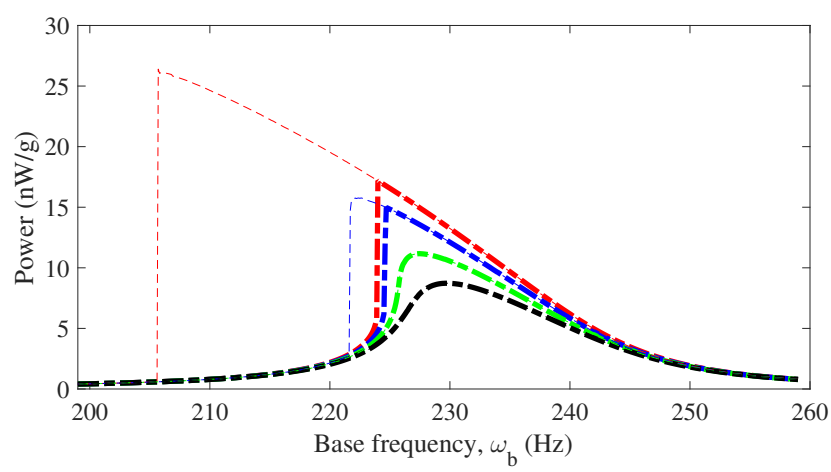

(d) Normalized Power

Fig. 4: The response for a varying base-excitation frequency $\left(a_{b}=0.2 \mathrm{~g}\right)$. Increasing the air damping $\left(c_{a}\right)$ qualitatively and quantitatively modifies the response. ( $c_{a}=0$ (red), 15 (blue), 30 (green) and $45 \mathrm{Ns}^{2} / \mathrm{m}^{2}$ (black)).

Figure 8. Mathematically, the modified governing equations are described by adding $c_{g} q(t) \dot{q}(t)^{2}$ and $c_{g} q(t) \dot{q}(t)^{2}$ to the right hand side of the governing equations (18)(19) where $c_{g}$ represents the control gain. To compute control power, we note that control power is equal to

Power $_{\text {control }}=\frac{1}{\Delta T} \int_{T_{1}}^{T_{2}} w(L, t)_{, t} G w(L, t) w(L, t)_{, t}^{2} \mathrm{~d} t$

$$
=G \psi^{4} \frac{1}{\Delta T} \int_{T_{1}}^{T_{2}} \dot{q}(t) q(t) \dot{q}(t)^{2} \mathrm{~d} t
$$

where $\Delta T=T_{2}-T_{1}$. And, the modal control gain is equal $c_{g}=\psi^{3} G$. Varying the modal control gain and setting the gain equal to $0,3 \times 10^{8}, 6 \times 10^{8}$, and $10 \times 10^{8}$ we compute the response of the closed-loop system and plot the tip displacement amplitude, the largest stable and unstable orbits, power spectrum of for $c_{g}=10 \times 10^{8}, \omega_{b}=198.65 \mathrm{~Hz}$, the averaged harvested power, and the averaged consumed mechanical control power shown in Figures 9a-9h. Note that the red curves show the result of open-loop (uncontrolled) response. To avoid subtracting power, the absolute terms are used to compute power, therefore, $\mid$ Power $_{\text {control }} \mid=$ $G \psi^{4} \frac{1}{\Delta T} \int_{T_{1}}^{T_{2}}|\dot{q}(t)||q(t)| \dot{q}(t)^{2} \mathrm{~d} t$. The harvested power is increased up to $191.1 \mathrm{nW}$ from the stable branch and up to $463.5 \mathrm{nW}$ from the unstable branch by expending up to $20.94 \mathrm{nW}$ and $36.14 \mathrm{nW}$ control power.

Increasing the nonlinear feedback gain, the frequencyresponse curve bends to the left (the backbone curve further moves downward), Figure 9a, and further limits the control power, Figure 9h. Therefore, using a larger gain is of value for larger base excitations. Changing the sign of control signal, response is computed and plotted in Figures 10a-10d. The hardening behaviour would further increase the harvested power and provide an opportunity to harvest up to an order of magnitude higher than the positive feedback.

\section{Conclusions}

A cantilever bimorph structure has been investigated to study the advantages of incorporating a control DC voltage and a nonlinear feedback on system performance. Static curves showed two unstable branches and a sta- 


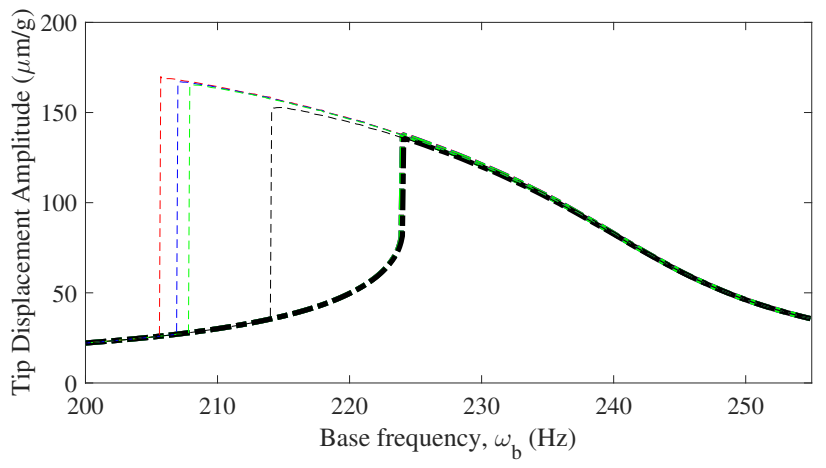

(a) Tip Displacement Amplitude

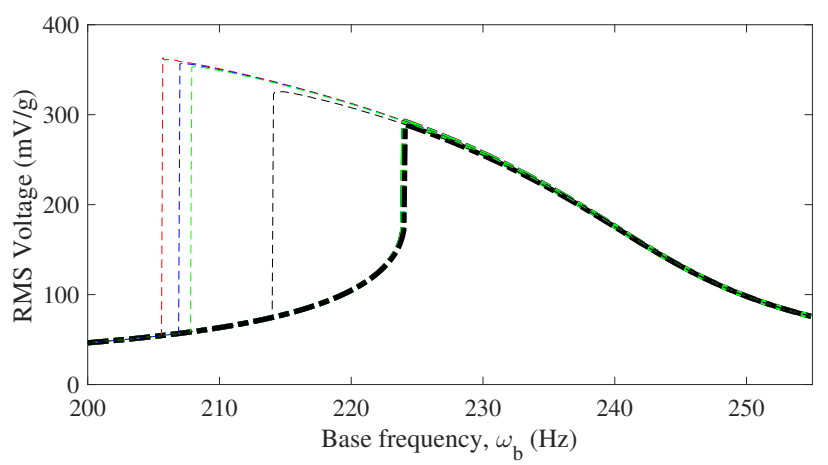

(c) Normalized Voltage

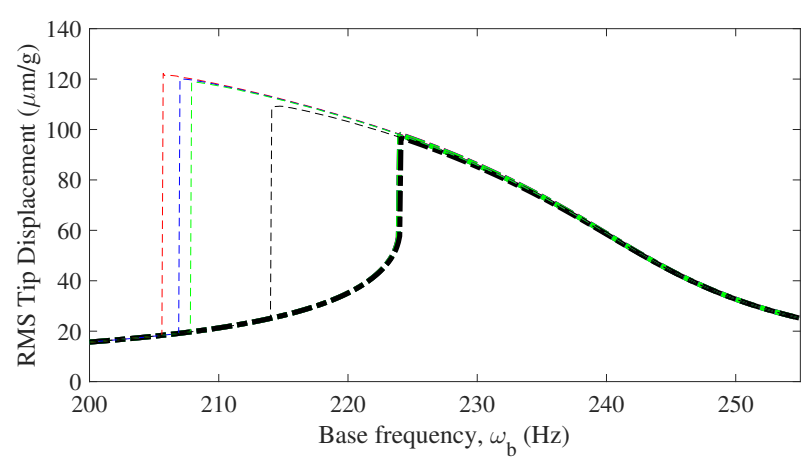

(b) Phase Portrait at $\omega_{b}=205.7 \mathrm{~Hz}$

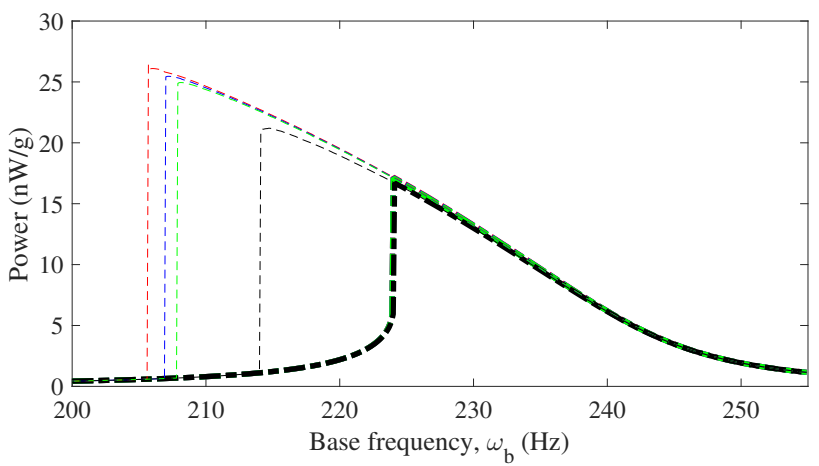

(d) Normalized Power

Fig. 5: The response for a varying base-excitation frequency $\left(a_{b}=0.2 \mathrm{~g}\right)$. Increasing the nonlinear damping $\left(c_{n}\right)$ affects the response. $\left(c_{n}=0\right.$ (red), $5 \times 10^{9}$ (blue), $10 \times 10^{9}$ (green) and $5 \times 10^{10} \mathrm{Ns} / \mathrm{m}^{3}$ (black)).

ble branch intersect at a subcritical pitchfork bifurcation point. The effective natural frequency curve was generated to help the designers find the desired operating point based on the application requirement. The DC voltage is used to tune the resonance frequency of structure and offer a potentially adaptable harvester to widen the range of optimum operation. The DC voltage may also be used to restrict the maximum dynamic response and reduce the probability of device failure. Varying nonlinear and air-damping, nonlinear material damping, the harvesting circuit parameters, and the external excitation, we offer a general framework for achieving the desired performance.

Furthermore, through designing a nonlinear feedback loop we have significantly improved the bandwidth and performance of the harvester and achieve a high level of harvested power without sacrificing the bandwidth. Using negative gains is advantageous over positive gains, and hardening behaviour is accompanied with high level of harvested power and large bandwidth. In the future, we will study the parallel connection case and further develop an averaging model of the system to provide a clear understanding of the vari- ous parameters. Furthermore, other possible excitation mechanisms and feedback loops are under investigation. 


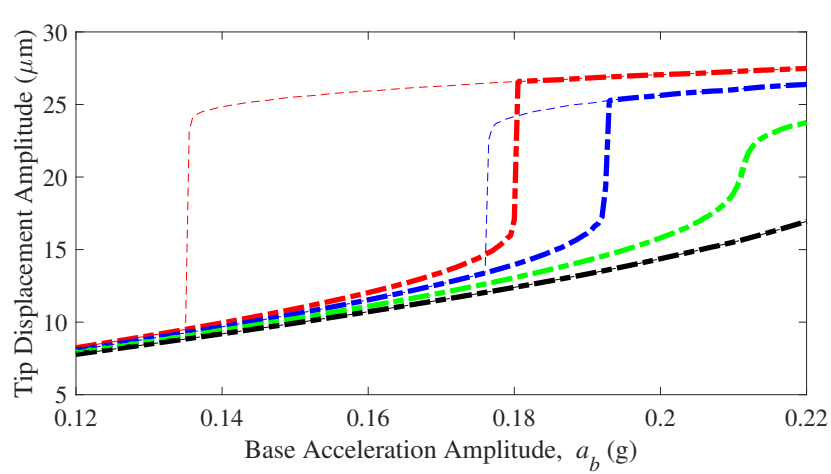

(a) Tip Displacement Amplitude

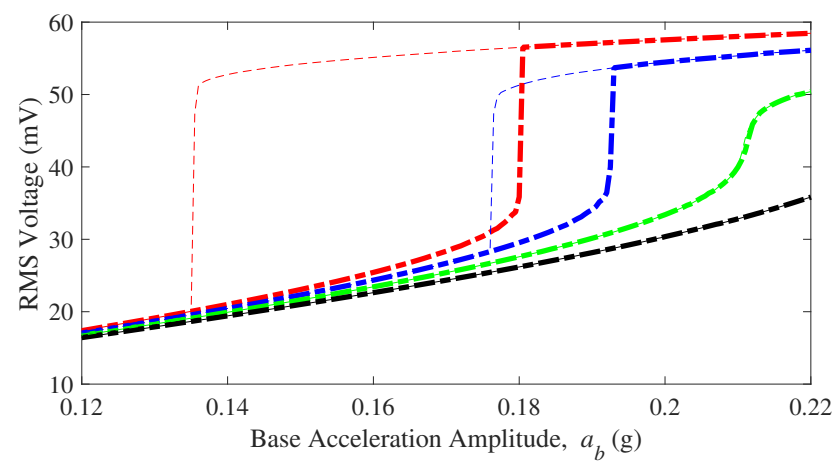

(c) Voltage

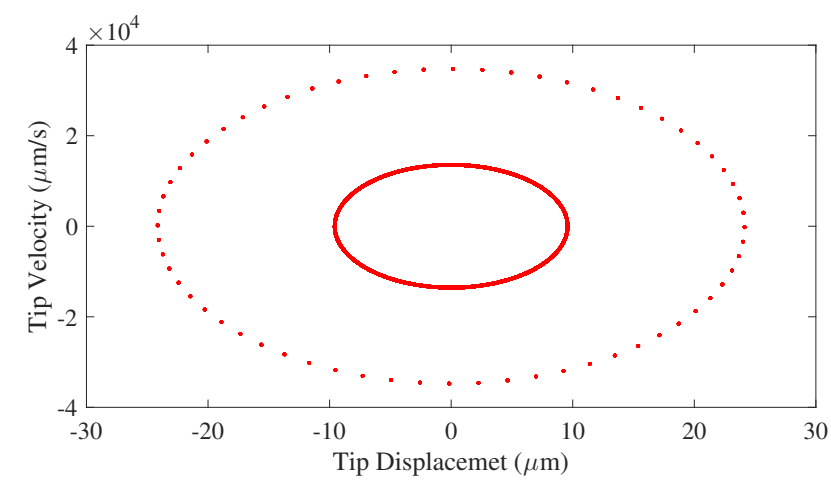

(e) Phase Portrait for $c_{a}=0 \mathrm{Ns}^{2} / \mathrm{m}^{2}$ at $a_{b}=0.136 \mathrm{~g}$

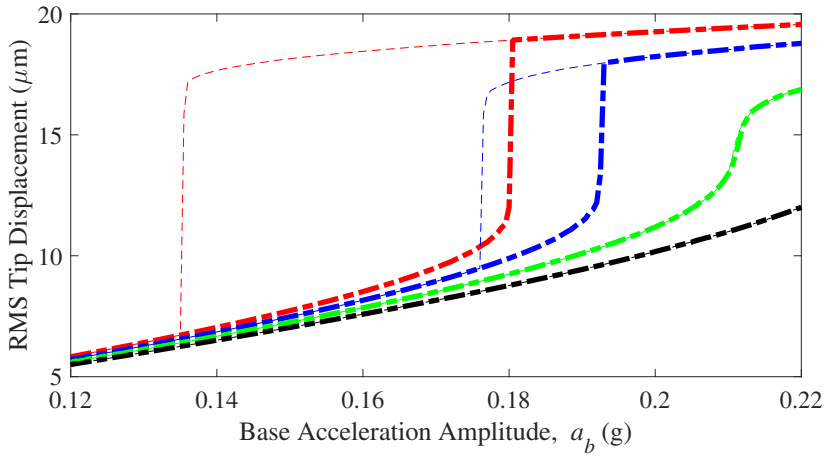

(b) RMS Tip Displacement

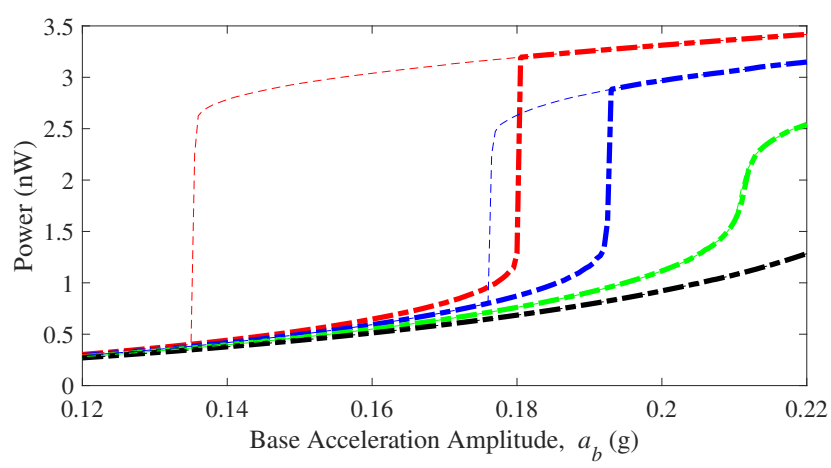

(d) Power

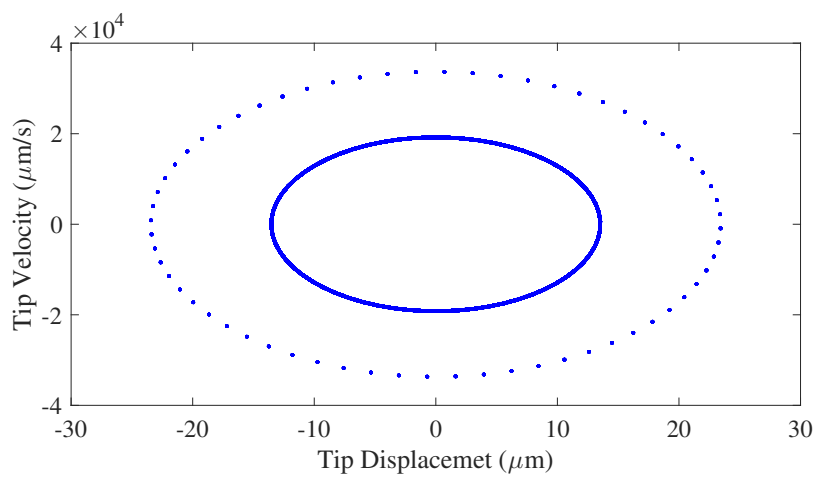

(f) Phase Portrait for $c_{a}=15 \mathrm{Ns}^{2} / \mathrm{m}^{2}$ at $a_{b}=0.177 \mathrm{~g}$

Fig. 6: The response for a varying base-acceleration. Increasing the air damping $\left(c_{a}\right)$ qualitatively and quantitatively modifies the response $\left(\omega_{b}=225 \mathrm{~Hz}\right) .\left(c_{a}=0\right.$ (red), 15 (blue), 30 (green) and $45 \mathrm{Ns}^{2} / \mathrm{m}^{2}$ (black)). 


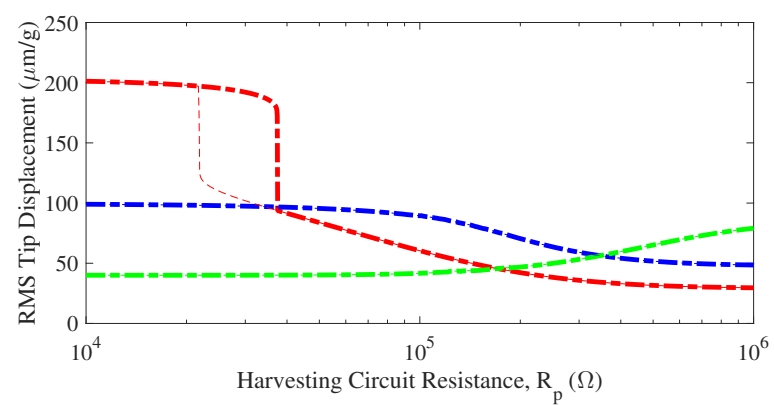

(a) Normalized RMS Tip Displacement

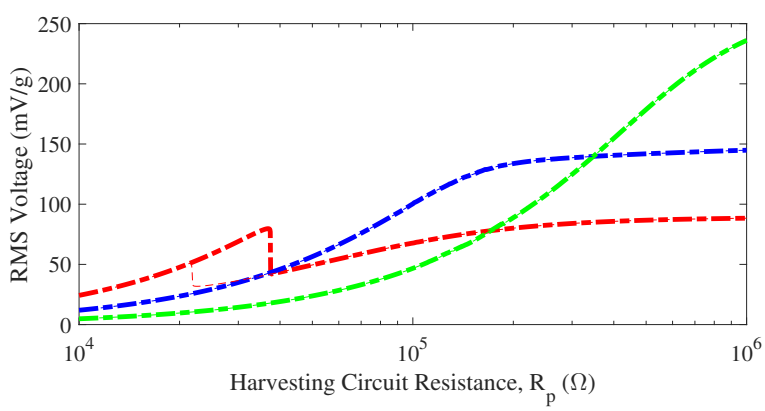

(b) Normalized RMS Voltage

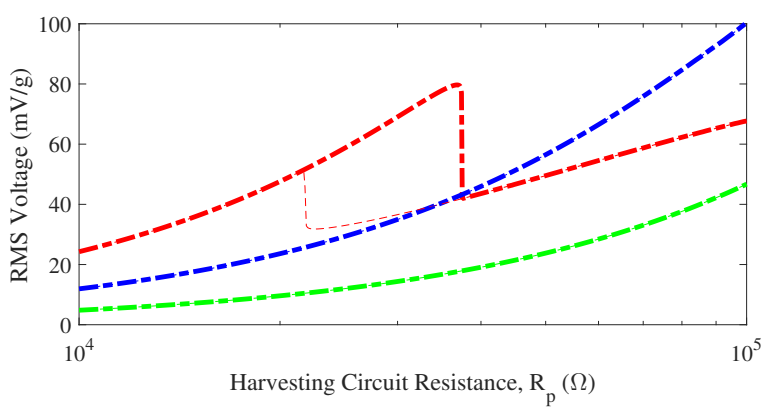

(c) Normalized RMS Voltage

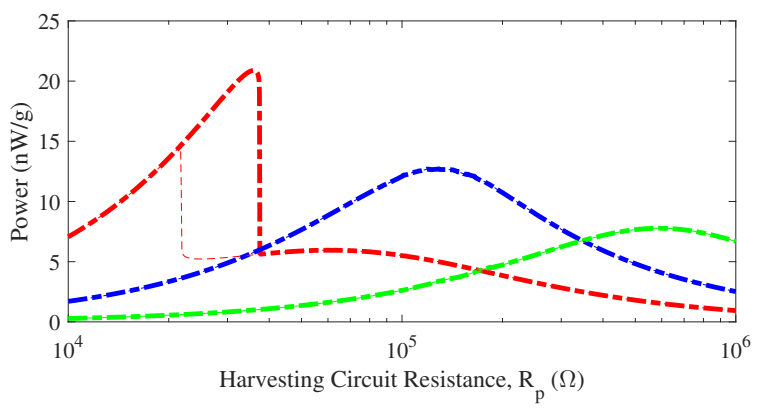

(d) Normalized Power

Fig. 7: The response, harvested power, and voltage versus the resistance of the harvesting circuit and the DC bias voltage $\left(a_{b}=0.12 \mathrm{~g}, \omega_{b}=225 \mathrm{~Hz}\right)$. ( $V_{\mathrm{es}}=5$ (red), 10 (blue), and $15 V$ (green) ).

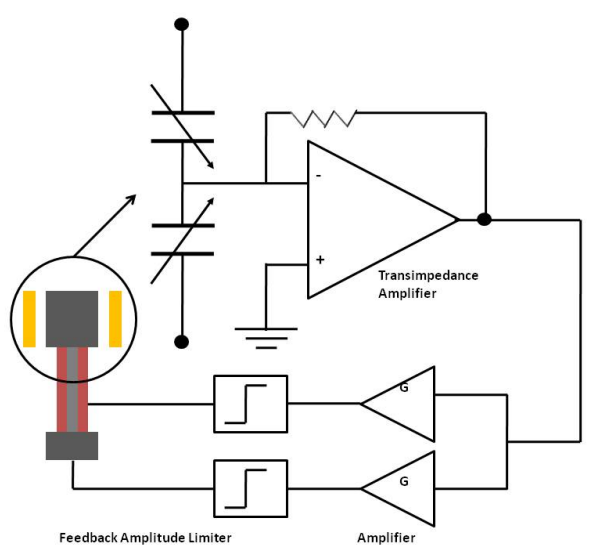

Fig. 8: Feedback loop to enhance the performance of the harvester. The figure is not drawn to scale. 


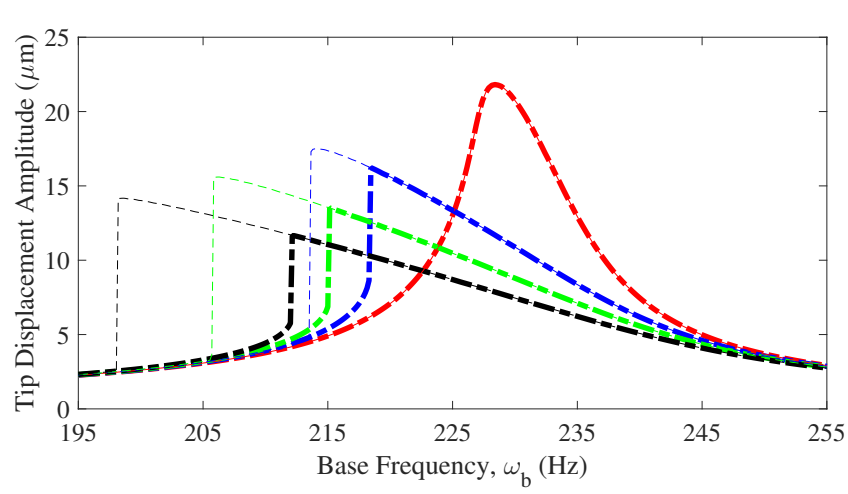

(a) Tip Displacement

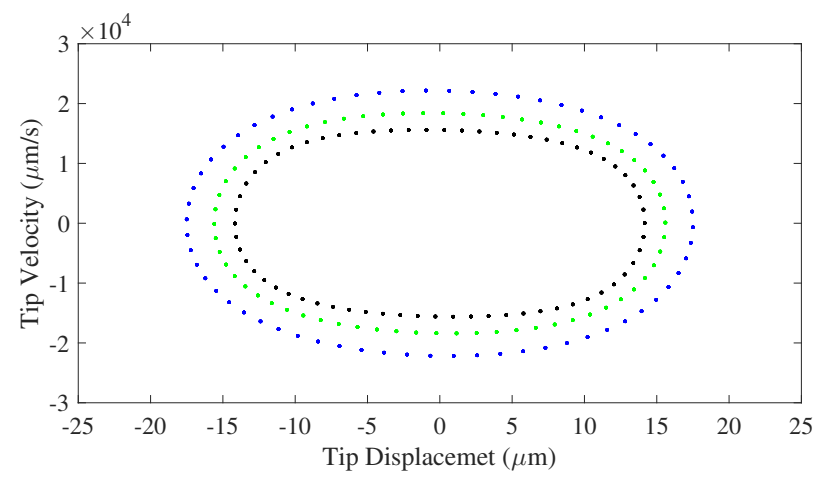

(c) Phase Portrait: Largest Unstable Orbits

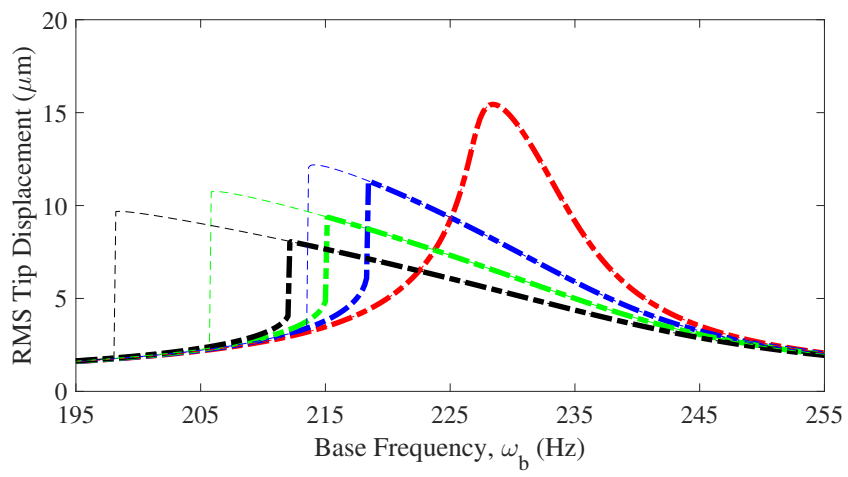

(e) RMS Tip Displacement

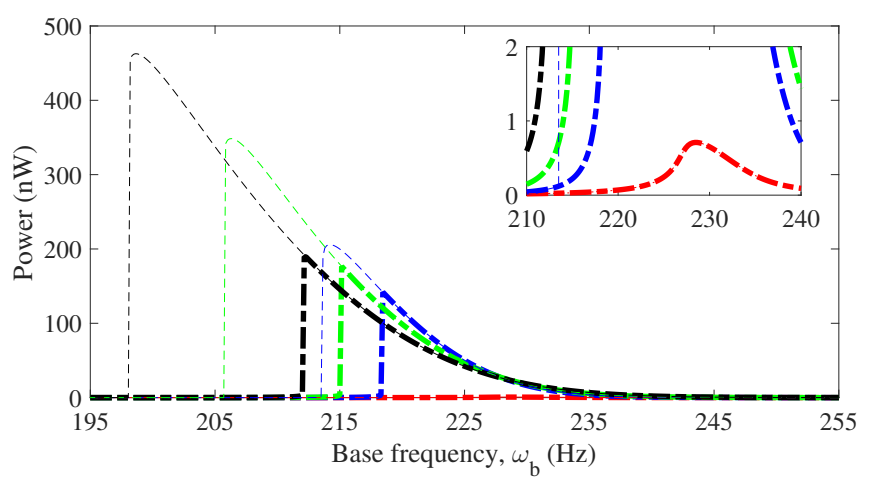

(g) Harvested Power

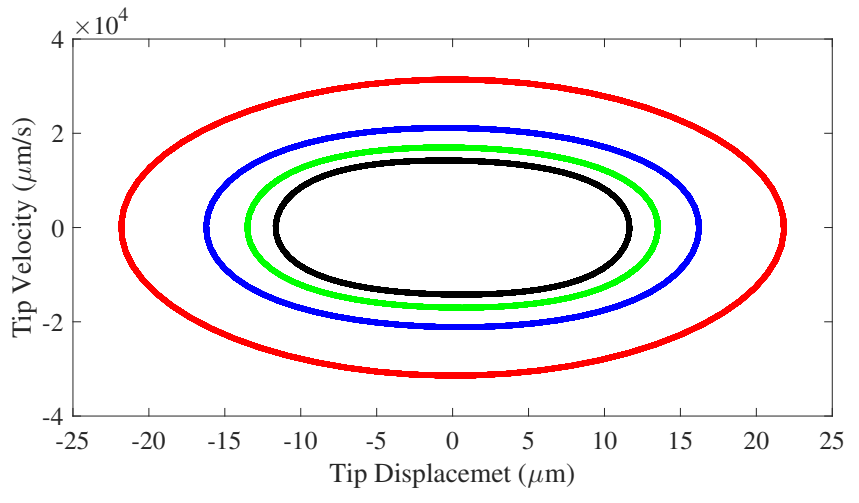

(b) Phase Portrait: Largest Stable Orbits

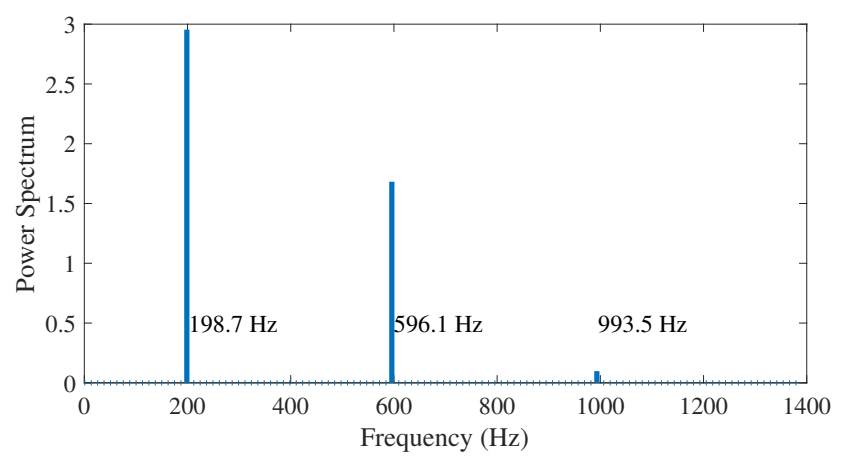

(d) Power Spectrum for $c_{g}=10 \times 10^{8}, \omega_{b}=198.65 \mathrm{~Hz}$

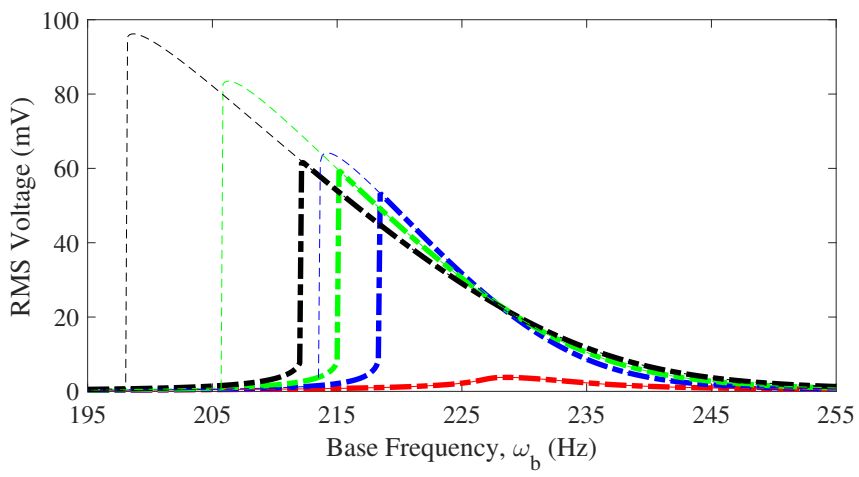

(f) RMS Output Voltage

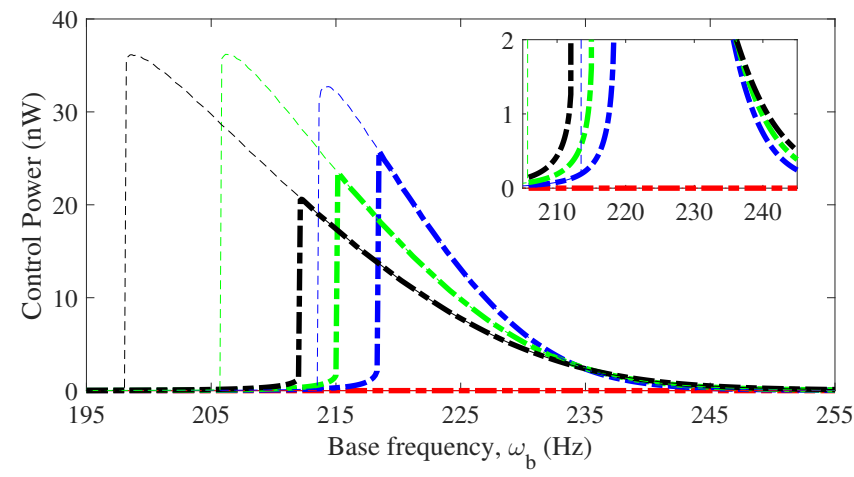

(h) Control Power

Fig. 9: The effect of the positive nonlinear feedback mechanism: the displacement response and the average harvested power for varying base frequency $\left(a_{b}=0.1 \mathrm{~g}, R_{p}=20 \mathrm{k} \Omega, V_{\mathrm{es}}=5 \mathrm{~V}, c_{a}=15\right)$. ( $c_{g}=0$ (red), $3 \times 10^{8}$ (blue), $6 \times 10^{8}$ (green), and $10 \times 10^{8}$ (black) $)$. 


\section{Appendix: Parameter Expressions}

In the following equations, prime ()$^{\prime}$ represents the spatial derivative,

$$
\begin{aligned}
& k_{l}=E_{s} I_{s} \int_{0}^{L_{s}} \psi^{\prime \prime}(r)^{2} \mathrm{~d} r \\
& k_{n}=E_{s} I_{s} \int_{0}^{L_{s}} \psi^{\prime}(r)^{2} \psi^{\prime \prime}(r)^{2} \mathrm{~d} r \\
& m_{l}=m_{p} \int_{0}^{L_{p}} \psi(r)^{2} d r+m_{s} \int_{0}^{L_{s}} \psi(r)^{2} \mathrm{~d} r \\
& +m_{t} \psi\left(L_{s}\right)^{2}+J_{t} \psi^{\prime}\left(L_{s}\right)^{2} \\
& m_{n}=m_{t}\left(\int_{0}^{L_{s}} \psi^{\prime}(r)^{2} \mathrm{~d} r\right)^{2}+J_{t} \psi^{\prime}\left(L_{s}\right)^{4} \\
& +m_{p} \int_{0}^{L_{p}}\left(\int_{0}^{r} \psi^{\prime}(r)^{2} d r\right)^{2} \mathrm{~d} r \\
& +m_{s} \int_{0}^{L_{s}}\left(\int_{0}^{r} \psi^{\prime}(r)^{2} \mathrm{~d} r\right)^{2} \mathrm{~d} r \\
& f_{l}=m_{p} \int_{0}^{L_{p}} \psi(r) z(r) d r+m_{s} \int_{0}^{L_{s}} \psi(r) z(r) \mathrm{d} r \\
& +m_{t} \psi\left(L_{s}\right) z\left(L_{s}\right) \\
& f_{n}=m_{t} z\left(L_{s}\right)^{2}+m_{p} \int_{0}^{L_{p}} z(r)^{2} \mathrm{~d} r \\
& +m_{s} \int_{0}^{L_{s}} z(r)^{2} \mathrm{~d} r \\
& h_{l}^{q}=\frac{1}{12} b c_{p}^{11} h_{p}\left(4 h_{p}^{2}+6 h_{p} h_{s}+3 h_{s}^{2}\right) \int_{0}^{L_{p}} \psi^{\prime \prime}(r)^{2} \mathrm{~d} r \\
& h_{l}^{\lambda}=-\frac{1}{2} b e_{31}\left(h_{p}+h_{s}\right) \int_{0}^{L_{p}} \psi^{\prime \prime}(r) \mathrm{d} r \\
& h_{n}^{\lambda}=-\frac{1}{4} b e_{31}\left(h_{p}+h_{s}\right) \int_{0}^{L_{p}} \psi^{\prime}(r)^{2} \psi^{\prime \prime}(r) \mathrm{d} r \\
& -\frac{1}{24} b e_{3111}\left(2 h_{p}^{3}+4 h_{p}^{2} h_{s}+3 h_{p} h_{s}^{2}+h_{s}^{3}\right) \\
& \times \int_{0}^{L_{p}} \psi^{\prime \prime}(r)^{3} \mathrm{~d} r \\
& h_{n}^{q}=\frac{1}{12} b c_{p}^{11} h_{p}\left(4 h_{p}^{2}+6 h_{p} h_{s}+3 h_{s}^{2}\right) \int_{0}^{L_{p}} \psi^{\prime}(r)^{2} \psi^{\prime \prime}(r)^{2} \mathrm{~d} r \\
& +\frac{1}{160} b c_{p}^{1111} h_{p} \\
& \times\left(16 h_{p}^{4}+40 h_{p}^{3} h_{s}+40 h_{p}^{2} h_{s}^{2}+20 h_{p} h_{s}^{3}+5 h_{s}^{4}\right) \\
& \times \int_{0}^{L_{p}} \psi^{\prime \prime}(r)^{4} \mathrm{~d} r \\
& C_{p}=-\frac{b L_{p} \epsilon_{33}}{4 h_{p}} \\
& \gamma_{l}=\frac{\epsilon_{0} \epsilon_{r}}{g^{3}}\left(2 b_{s}+0.33125\left(b_{s}^{0.25}+h_{s}^{0.25}\right) g^{0.75}\right) \\
& \times \int_{0}^{L_{m}}\left(\psi\left(L_{s}\right)+r \psi^{\prime}\left(L_{s}\right)\right)^{2} \mathrm{~d} r \\
& \gamma_{n}=\frac{\epsilon_{0} \epsilon_{r}}{g^{5}}\left(2 b_{s}+0.201856\left(b_{s}^{0.25}+h_{s}^{0.25}\right) g^{0.75}\right) \\
& \times \int_{0}^{L_{m}}\left(\psi\left(L_{s}\right)+r \psi^{\prime}\left(L_{s}\right)\right)^{4} \mathrm{~d} r
\end{aligned}
$$

Conflict of interest. The authors declare that they have no conflict of interest. Funding. Authors have not receive any funding for this study. Authors have no sponsor for this study.

\section{References}

1. Abdelkefi, A., Nayfeh, A., Hajj, M.: Global nonlinear distributed-parameter model of parametrically excited piezoelectric energy harvesters. Nonlinear Dynamics 67(2), 1147-1160 (2012)

2. Alevras, P., Theodossiades, S., Rahnejat, H.: On the dynamics of a nonlinear energy harvester with multiple resonant zones. Nonlinear Dynamics pp. 1-16 (2018)

3. Arnold, D.P.: Review of microscale magnetic power generation. IEEE Transactions on Magnetics 43(11), 39403951 (2007)

4. Beeby, S.P., Tudor, M.J., White, N.: Energy harvesting vibration sources for microsystems applications. Measurement science and technology 17(12), R175 (2006)

5. Chen, C., Zanette, D.H., Guest, J.R., Czaplewski, D.A., López, D.: Self-sustained micromechanical oscillator with linear feedback. Physical review letters 117(1), 017203 (2016)

6. Cook-Chennault, K., Thambi, N., Sastry, A.: Powering mems portable devicesa review of non-regenerative and regenerative power supply systems with special emphasis on piezoelectric energy harvesting systems. Smart Materials and Structures 17(4), 043001 (2008)

7. Daqaq, M.F., Stabler, C., Qaroush, Y., Seuaciuc-Osorio, T.: Investigation of power harvesting via parametric excitations. Journal of Intelligent Material Systems and Structures 20(5), 545-557 (2009)

8. Du Toit, N.E.: Modeling and design of a mems piezoelectric vibration energy harvester (2005). Master's thesis, Massachusetts Institute of Technology (MIT)

9. Ferrari, M., Ferrari, V., Guizzetti, M., Andò, B., Baglio, S., Trigona, C.: Improved energy harvesting from wideband vibrations by nonlinear piezoelectric converters. Sensors and Actuators A: Physical 162(2), 425-431 (2010)

10. Glynne-Jones, P., Beeby, S.P., White, N.M.: Towards a piezoelectric vibration-powered microgenerator. IEE Proceedings - Science, Measurement and Technology 148(2), 68-72 (2001)

11. Hu, Y.C., Chang, P.Z., Chuang, W.C.: An approximate analytical solution to the pull-in voltage of a micro bridge with an elastic boundary. Journal of Micromechanics and Microengineering 17(9), 1870 (2007)

12. Hwang, M., Arrieta, A.F.: Input-independent energy harvesting in bistable lattices from transition waves. Scientific reports 8(1), 3630 (2018)

13. Jeon, Y., Sood, R., Jeong, J.H., Kim, S.G.: MEMS power generator with transverse mode thin film pzt. Sensors and Actuators A: Physical 122(1), 16-22 (2005)

14. Jia, Y., Seshia, A.A.: An auto-parametrically excited vibration energy harvester. Sensors and Actuators A: Physical 220, 69-75 (2014)

15. Jia, Y., Seshia, A.A.: Five topologies of cantilever-based MEMS piezoelectric vibration energy harvesters: a numerical and experimental comparison. Microsystem Technologies 22(12), 2841-2852 (2016)

16. Jia, Y., Yan, J., Soga, K., Seshia, A.A.: Parametric resonance for vibration energy harvesting with design techniques to passively reduce the initiation threshold amplitude. Smart Materials and Structures 23(6), 065011 (2014) 


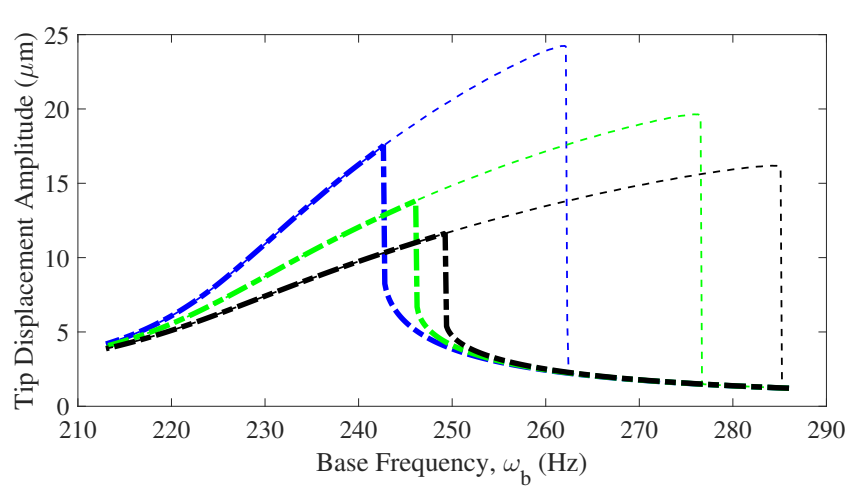

(a) Tip Displacement

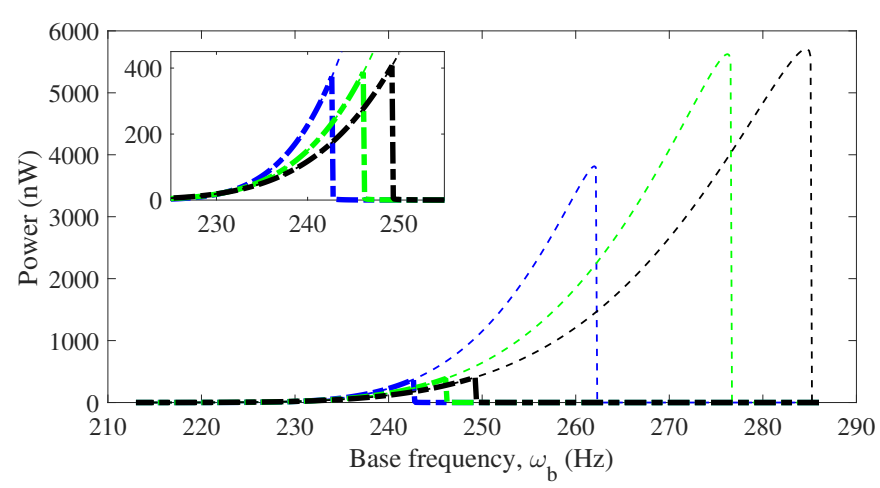

(c) Harvested Power

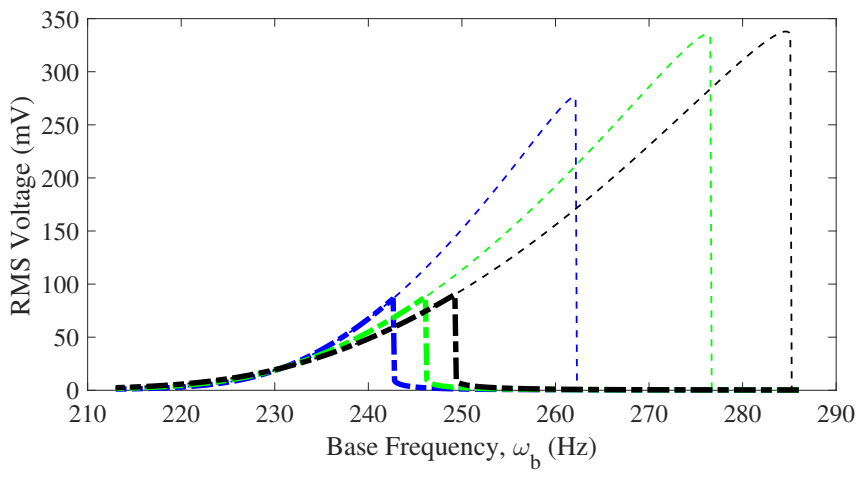

(b) RMS Output Voltage

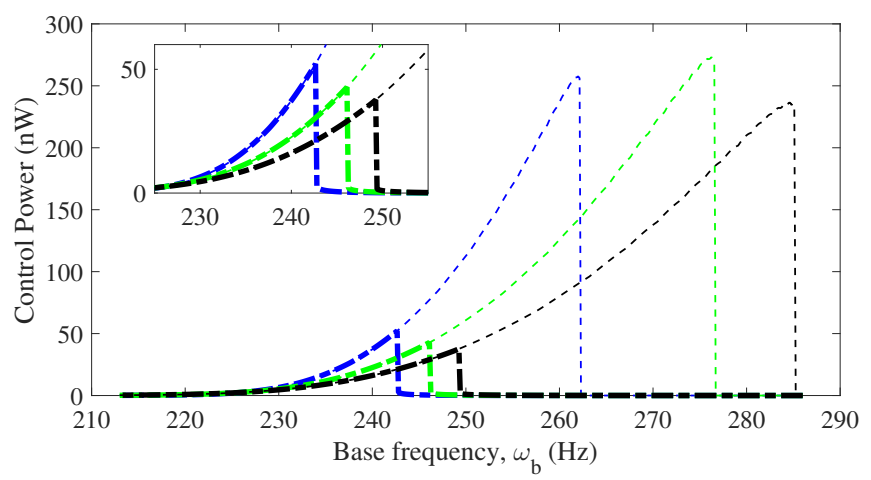

(d) Control Power

Fig. 10: The effect of the positive nonlinear feedback mechanism: the displacement response and the average harvested power for varying base frequency $\left(a_{b}=0.1 \mathrm{~g}, R_{p}=20 \mathrm{k} \Omega, V_{\mathrm{es}}=5 \mathrm{~V}, c_{a}=15\right)$. $\left(c_{g}=-3 \times 10^{8}\right.$ (blue), $-6 \times 10^{8}$ (green), and $-10 \times 10^{8}$ (black) ).

17. Kumar, A., Ali, S.F., Arockiarajan, A.: Exploring the benefits of an asymmetric monostable potential function in broadband vibration energy harvesting. Applied Physics Letters 112(23), 233901 (2018)

18. Lajimi, S.A.M.: Apparatus and method for improved shock, impact, and motion detection (2019). US Patent App. $62 / 839,378$

19. Lajimi, S.A.M., Heppler, G.R.: Free vibration and buckling of cantilever beams under linearly varying axial load carrying an eccentric end rigid body. Transactions of the Canadian Society for Mechanical Engineering 37(1), 89109 (2013)

20. Lajimi, S.A.M., Noori, N., Marzouk, A., Bahreyni, B., Golnaraghi, F.: A novel nonlinear amplitude-modulation gyroscope incorporating internal resonance. In: Proceedings of the 25th CANCAM, pp. 1-4 (2015)

21. Leadenham, S., Erturk, A.: M-shaped asymmetric nonlinear oscillator for broadband vibration energy harvesting: Harmonic balance analysis and experimental validation. Journal of Sound and Vibration 333(23), 6209-6223 (2014)

22. Leadenham, S., Erturk, A.: Unified nonlinear electroelastic dynamics of a bimorph piezoelectric cantilever for energy harvesting, sensing, and actuation. Nonlinear Dynamics 79(3), 1727-1743 (2015)

23. Lee, H., Sharpes, N., Abdelmoula, H., Abdelkefi, A., Priya, S.: Higher power generation from torsion- dominant mode in a zigzag shaped two-dimensional energy harvester. Applied Energy 216, 494-503 (2018)

24. Liu, D., Al-Haik, M., Zakaria, M., Hajj, M.R.: Piezoelectric energy harvesting using l-shaped structures. Journal of Intelligent Material Systems and Structures 29(6), 1206-1215 (2018)

25. Madinei, H., Khodaparast, H.H., Adhikari, S., Friswell, M.: Design of MEMS piezoelectric harvesters with electrostatically adjustable resonance frequency. Mechanical Systems and Signal Processing 81, 360-374 (2016)

26. Madinei, H., Khodaparast, H.H., Friswell, M., Adhikari, S.: Minimising the effects of manufacturing uncertainties in mems energy harvesters. Energy 149, 990-999 (2018)

27. van der Meijs, N., Fokkema, J.: VLSI circuit reconstruction from mask topology. INTEGRATION, The VLSI Journal 2(2), 85-119 (1984)

28. Meirovitch, L.: Principles and techniques of vibrations, vol. 1. Prentice Hall New Jersey (1997)

29. Mitcheson, P.D., Miao, P., Stark, B.H., Yeatman, E., Holmes, A., Green, T.: Mems electrostatic micropower generator for low frequency operation. Sensors and Actuators A: Physical 115(2-3), 523-529 (2004)

30. Mousavi Lajimi, S.A., Friswell, M.I.: Energy harvesting from a non-linear standing beam-mass system: Twoversus one-mode approximations. Journal of Intelligent Material Systems and Structures 28(8), 1010-1022 (2017) 
31. Nayfeh, A.H., Pai, P.F.: Non-linear non-planar parametric responses of an inextensional beam. International Journal of Non-Linear Mechanics 24(2), 139-158 (1989)

32. Osterberg, P.M., Senturia, S.D.: M-TEST: a test chip for MEMS material property measurement using electrostatically actuated test structures. Journal of Microelectromechanical Systems 6(2), 107-118 (1997)

33. Ramini, A., Alcheikh, N., Ilyas, S., Younis, M.I.: Efficient primary and parametric resonance excitation of bistable resonators. AIP Advances 6(9), 095307 (2016)

34. Ramini, A.H., Hajjaj, A.Z., Younis, M.I.: Tunable resonators for nonlinear modal interactions. Scientific reports 6, 34717 (2016)

35. Ramini, A.H., Hennawi, Q.M., Younis, M.I.: Theoretical and experimental investigation of the nonlinear behavior of an electrostatically actuated in-plane mems arch. Journal of Microelectromechanical Systems 25(3), 570$578(2016)$

36. Crespo da Silva, M., Glynn, C.: Nonlinear flexuralflexural-torsional dynamics of inextensional beams. i. equations of motion. Journal of Structural Mechanics 6(4), 437-448 (1978)

37. Stanton, S.C., Erturk, A., Mann, B.P., Dowell, E.H., Inman, D.J.: Nonlinear nonconservative behavior and modeling of piezoelectric energy harvesters including proof mass effects. Journal of Intelligent Material Systems and Structures 23(2), 183-199 (2012)

38. Stanton, S.C., McGehee, C.C., Mann, B.P.: Reversible hysteresis for broadband magnetopiezoelastic energy harvesting. Applied Physics Letters 95(17), 174103 (2009)

39. Stanton, S.C., McGehee, C.C., Mann, B.P.: Nonlinear dynamics for broadband energy harvesting: Investigation of a bistable piezoelectric inertial generator. Physica D: Nonlinear Phenomena 239(10), 640-653 (2010)

40. Wang, Q.M., Cross, L.E.: Constitutive equations of symmetrical triple layer piezoelectric benders. IEEE transactions on Ultrasonics, Ferroelectrics, and Frequency Control 46(6), 1343-1351 (1999)

41. Ya'akobovitz, A., Krylov, S.: Toward sensitivity enhancement of mems accelerometers using mechanical amplification mechanism. IEEE sensors journal 10(8), 1311-1319 (2010)

42. Yang, Z., Zhou, S., Zu, J., Inman, D.: High-performance piezoelectric energy harvesters and their applications. Joule pp. 642-697 (2018) 